\title{
A Molecular Dynamics Simulation of the Orientationaly Disor- dered Phase of Potassium Perchlorate
}

\author{
F. Affouard $\left({ }^{*}\right)$ and Ph. Depondt \\ Département de Recherches Physiques( ${ }^{* *}$ ), Université Pierre et Marie Curie, 4. place Jussieu, \\ 75252 Parss Cedex 05 France
}

(Recerved 13 July 1995. accepted in final form 21 September 1995)

\begin{abstract}
The orientationaly disordered phase of a relatively large system of potassium perchlorate is simulated on a massively parallel computer with strict SIMD architecture. Crystallographic results are compared with known diffraction data. Correlation densities of orientational and translational variables are computed showing orientation-translation coupling of the perchlorate ions, and coupling between a perchlorate orrentation and the translation of neighbouring potassium and perchlorate ions. The orlentation-orientation correlation between neighbouring perchlorate lons shows antiferromagnetic pseudo-spin type coupling corresponding to ions in opposite $T_{\mathrm{d}}$ orientations. This correlation has a lifetime which is longer than the single molecule orientational lifetime, evidence of correlated orlentational motion.
\end{abstract}

PACS. $61.12-q$ - Neutron diffraction and scattering.

PACS. 61.43-j - Disordered solıds.

PACS. 61.43Bn - Structural modeling: serıal-addition models, computer simulation.

\section{Introduction}

Potassium perchlorate $\mathrm{KClO}_{4}$ exhibits at approximately $T=310^{\circ} \mathrm{C}$ a transition from an orthorhombic [1] ( $\mathrm{D}_{2 \mathrm{~h}}^{16}$ - Pnma) phase to a fcc [2] $\left(\mathrm{O}_{\mathrm{h}}^{5}-\mathrm{Fm} 3 \mathrm{~m}\right)$ structure in which the perchlorate ıons are orientationaly disordered. Neutron diffraction experiments [3] performed on a single crystal of the high temperature phase at $325^{\circ} \mathrm{C}$ and $420^{\circ} \mathrm{C}$, showed that the $\mathrm{ClO}_{4}^{-}$ions have preferred orlentations of $T_{\mathrm{d}}$ symmetry with orientational disorder (Fig. 1). Such plastic crystals are highly disordered but retain the long range order of the molecular centers of mass thus allowing experiments such as diffraction that would otherwise be impossible. However these experıments are often difficult to interpret, the relevant information being partially smeared out by disorder. A Molecular Dynamics computer simulation (MD) [4], on the other hand, ylelds extensive information on the simulated system, enabling comparisons with experimental results and precise descriptions.

The present paper presents a simulation of potassium perchlorate in the disordered phase, similar to that in reference [5]. However, the most computer time and memory consuming part in a simulation is the parr force calculation which limits the number of molecules in the simulation to approximately 1000 in most practical cases, and the simulation duration to approximately 100 picoseconds. In many situations, one may wish larger samples for e.g.

(*) Present address: Institut für theoretische Physık, Technische Universıtät Berlın

$\left({ }^{* *}\right)$ URA CNRS 71

(C) Les Éditions de Physıque 1996 


$$
\sigma=1
$$

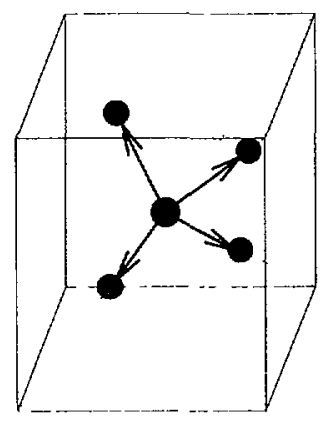

$0 \quad \mathrm{Cl}$ $\sigma=-1$

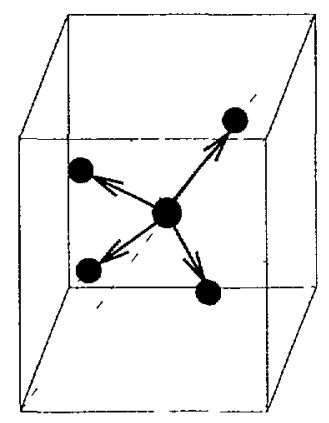

Fig. 1. - The two orientations of the perchlorate 1ons with $T_{d}$ symmetry.

long wavelength or small wavenumber studies, and longer durations for e.g. phase transition studies. The use of efficient programming methods, such as a cutoff radius or a neighbour list for short range interactions, an Ewald sum [6] for long range electrostatic forces or the cell multipole method [7] for clusters, can reduce CPU time to a certain extent. However, in order to increase significantly the size of the system, one has to use more powerful computers, and that often translates into parallelism. Our simulation is therefore done on a SIMD (Single Instruction Multiple Data) massively parallel computer in order to be able to study ultimately phase transition dynamics for which all the computational power avallable is necessary.

In Section 2, some aspects of SIMD parallelism are discussed in relation to MD simulation, along with the description of our potassium perchlorate simulation. We compare in Section 3 our results with diffraction data [3] which was not available to the authors of reference [5]. In Section 4, following a procedure described in [8], we study the single molecule orientationtranslation (TO) correlations for the $\mathrm{ClO}_{4}$ ions, the TO correlations between a perchlorate and a neighbouring potassium ion, and between the translations of one perchlorate ion and the orientation of another. Finally, in Section 5, orientation-orientation (OO) correlations between two neighbouring perchlorate ions are discussed, from both a static and a dynamical point of view.

\section{The Simulation}

2.1. Parallelism. - There are several different types of parallel computers. For the purpose of MD simulations, transputer arrays, which run in MIMD (Multiple Instructions Multiple Data) mode have been used with success [9], and message-passing software such as PVM (Parallel Virtual Machine) also seems promising. Our molecular dynamics simulation was done on a CM2 Connection Machine, a massively parallel computer, with distributed memory; to our knowledge, such architecture was little used up to now for MD. The 16384 processors of the CM2 run in SIMD [10] mode, in which all the processors in use execute the same instruction at the same time using their local data. The $2^{n}$ processors are layed out on a $n$ - 
Table I. -- Simulation parameters.

\begin{tabular}{|c|c|c|c|c|}
\hline $\begin{array}{c}\text { time step } \\
\text { ps }\end{array}$ & $\begin{array}{c}\text { cutoff } \\
\AA\end{array}$ & $\begin{array}{c}\text { piston mass } \\
\text { amu }\end{array}$ & $\begin{array}{c}\text { Cl-O bond length } \\
\AA\end{array}$ & $\begin{array}{c}\text { external pressure } \\
\text { atm }\end{array}$ \\
\hline $5.10^{-3}$ & 8.5 & 300 & 1.42 & $\mathbf{1}$ \\
\hline
\end{tabular}

dimensional hypercube with implicit periodicity in each direction, each processor thus having $n$ neighbours. As in many parallel computing situations, communications between processors are the main problem. The two main communication mechanisms are NEWS (North, East, West, South) [11] very fast communications between neighbours on the grid of the hypercube, and general communications between non-neighbours, which are approximately four times slower.

The CM-FORTRAN compiler automatically allocates arrays to processors according to how the arrays are used in the program. The bulk of a MD simulation program consists of the calculation of the forces between particle pairs $i, j$, i.e. $\mathbf{f}\left(r_{\imath \jmath}\right)$, where $\mathbf{f}$ is the analytical form of the force between two particles at distance $r_{\imath \jmath}$ and $i$ and $j$ are neighbours. If the program can be written so that the compler can make neighbouring particles also to be neighbours on the hypercube, then NEWS communications take place. This implies 1) that the crystal structure remains stable throughout the simulation, and 2) that the number of neighbours is smaller than $2 n$, where $n$ is the dimensionality of the hypercube. Usually neither condition is met. Because of long-range electrostatic forces, the number of neighbours which must be included in the force calculation can be large, despite the use of Ewald summations. Our simulations are done with a constant number of particles, a constant pressure and a constant enthalpy, i.e. in the NPH ensemble [12], thus allowing the global shape of the system to change when reaching equilibrium, and we wish eventually to be able to simulate phase transitions, for which, clearly the number of neighbours is apt to change. The computer thus follows the nonoptimum general communication scheme. Nevertheless, using, to compute forces, neighbours lists defined by a cutoff radius, we were able to simulate large systems $(8 \times 8 \times 8$ cells, a total of $2048 \mathrm{KClO}_{4}$ unts i.e. 24576 force centers), in reasonable times: $\sim 7.5 \mathrm{~s}$ per step for 64 neighbours in the neighbour list, $\sim 4 \mathrm{~s}$ for 32 neighbours, with neighbour list reactualization every tenth step, despite the time-consuming long range electrostatic forces. Therefore, the sheer power of the $2^{n}$ processors compensates for the non-optimum communication situation.

2.2. The Simulation of Potassium Perchlorate. - The main parameters of the simulation (NPH ensemble with periodic boundary conditions, the perchlorate ions being consudered rigid) are summarized in Table I. To forbid the rotation of the cell as a whole and to simplify the analysis of the molecular orientations and the crystal structure, the antisymmetric part of the matrix that describes the shape of the cell (matrix $\mathbf{H}$ in [12]) is forced to zero at each step. The main parameters of the simulation are summarized in Table $\mathrm{I}$.

Our initial configuration is a perfect fcc structure for the positions, a temperature adapted Gaussian distribution for translational and angular velocities, and a unform distribution for the quaternions that describe the orientations of the perchlorate cons. Equilibrium is attained in approximately 5 picoseconds. The positions and the quaternions were saved every twenty steps (0.1 ps).

2.2.1. Short Range Interactions. - The atom-atom short range forces were computed within the cutoff radius given in Table I from a classical exp- 6 potential:

$$
V^{\mathrm{sr}}=\sum_{\imath, \alpha, \beta}\left(A_{\alpha \beta} \exp \left(-B_{\alpha \beta} r_{\imath \jmath \alpha \beta}\right)-\frac{C_{\alpha \beta}}{r_{\imath \jmath \alpha \beta}^{6}}\right)
$$


Table II. - Simulation coefficients for short range potential.

\begin{tabular}{|c|ccc|}
\hline$\alpha-\beta$ & $A_{\alpha \beta}\left(\mathrm{kJ} \mathrm{mol}^{-1}\right)$ & $B_{\alpha \beta}\left(\AA^{-1}\right)$ & $C_{\alpha \beta}\left(\mathrm{kJ} \mathrm{mol}^{-1}\left(\AA^{6}\right)\right)$ \\
\hline $\mathrm{K}-\mathrm{Cl}$ & 221545 & 3.14 & 3098 \\
$\mathrm{~K}-\mathrm{K}$ & 150100 & 2.97 & 1464 \\
$\mathrm{~K}-\mathrm{O}$ & 223185 & 3.52 & 1301 \\
$\mathrm{Cl}-\mathrm{Cl}$ & 327000 & 3.31 & 6554 \\
$\mathrm{O}-\mathrm{O}$ & 331850 & 4.07 & 1156 \\
$\mathrm{O}-\mathrm{Cl}$ & 329420 & 3.69 & 2753 \\
\hline
\end{tabular}

where $r_{\imath \jmath \alpha \beta}$ is the distance between atom $\alpha$ of molecule $i$ and atom $\beta$ of molecule $\jmath$ :

$$
r_{\imath \jmath \alpha \beta}=\left\|\mathbf{r}_{\imath \jmath \alpha \beta}\right\|=\left\|\mathbf{r}_{2}^{\alpha}-\mathbf{r}_{3}^{\beta}\right\|
$$

The values of the coefficients $A_{\alpha \beta}, B_{\alpha \beta}$ and $C_{\alpha \beta}$ (Tab. II) are the same as in [5].

2.2.2. Long Range Interactions. - With periodic boundary conditions, the simulated cell is replicated throughout space to form an infinite lattice. If $\mathbf{n}$ labels replicated cell of edge $L$, the total long range electrostatic potential is:

$$
V^{\prime r}=\sum_{\mathbf{n}} \sum_{2,3, \alpha, \beta} \frac{q_{\alpha} \cdot q_{\beta}}{\left\|\mathbf{r}_{2 j \alpha \beta}+\mathbf{n} L\right\|}
$$

where a cubic shaped cell is assumed for simplicity, and $q_{\alpha}$ is the charge localized on the atom $\alpha$ with the distribution: $q_{\mathrm{K}}=+e, q_{\mathrm{Cl}}=0, q_{\mathrm{O}}=-0.25 e$.

The Ewald sum $[6,12]$ yıelds :

$$
V^{\text {rewald }}=\sum_{\imath \jmath, \alpha, \beta} q_{\alpha} q_{\beta}\left(\frac{\operatorname{erfc}\left(\eta r_{\imath \jmath \alpha \beta}\right)}{r_{\imath \jmath \alpha \beta}}+\frac{2 \pi}{V} \sum_{\mathbf{q} \neq \mathbf{0}} \frac{1}{q^{2}} \exp \left(-\frac{q^{2}}{4 \eta^{2}}\right) \cos \left(\mathbf{q} \cdot \mathbf{r}_{\imath \jmath \alpha \beta}\right)-\frac{\eta}{\sqrt{\pi}}\right)
$$

where $\operatorname{erfc}(x)$ is the complementary error function, and $\eta$ a parameter that accelerates the convergence. The sum $\sum_{\imath_{3}, \alpha, \beta}$ ranges in principle over all the charges in the cell, but due to the rapid convergence of erfc( $x)$, the first term is limited to the same cutoff radius as the short range forces and thus can be treated similarly. The long range part of the potential is computed in reciprocal space using the periodicity of the replicated cells.

Potential $V^{\text {ewald }}$ gives a very good approximation of $V^{\mathrm{lr}}$ with a fast convergence if parameter $\eta$ is properly chosen. In order to choose the best value for $\eta$ [13], Figure 2 shows $V^{\text {ewald }}$ and $F^{\text {ewald }}=\sum\|\mathbf{f}\|^{2}$, the sum of squared forces, for different values of $\eta$ and the cutoff radius. We finally retained $\eta=\frac{16}{L}$, so that the results are fairly independent of both $\eta$ and of the cutoff radius.

\section{Comparison with Diffraction Experiments}

The simulations were done at two temperatures in the plastic phase. In both cases, the correct fcc structure was obtained. Figure 3 shows the orlentational probability density function $P(\Omega)$, where $\Omega$ denotes the orientation of a perchlorate ion 1.e. the rotation of Euler angles $\psi, \theta$ and $\phi$ that rotates the molecular reference frame unto the crystal frame. Given that the orientational origin is a $T_{\mathrm{d}}$ orientation in which the four molecular threefold axes are parallel to four of the elght crystal ones $([1 \overline{1} 1],[\overline{1} 11],[11 \overline{1}],[\overline{1} \overline{1}])$, Figure 3 shows peaks in the $T_{\mathrm{d}}$ orientations in 
a)

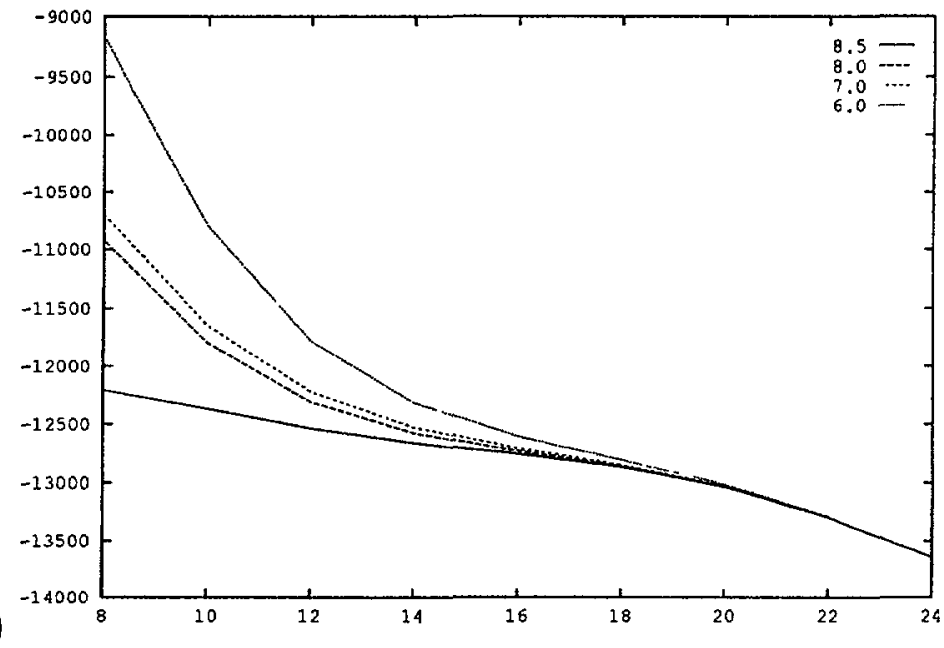

b)

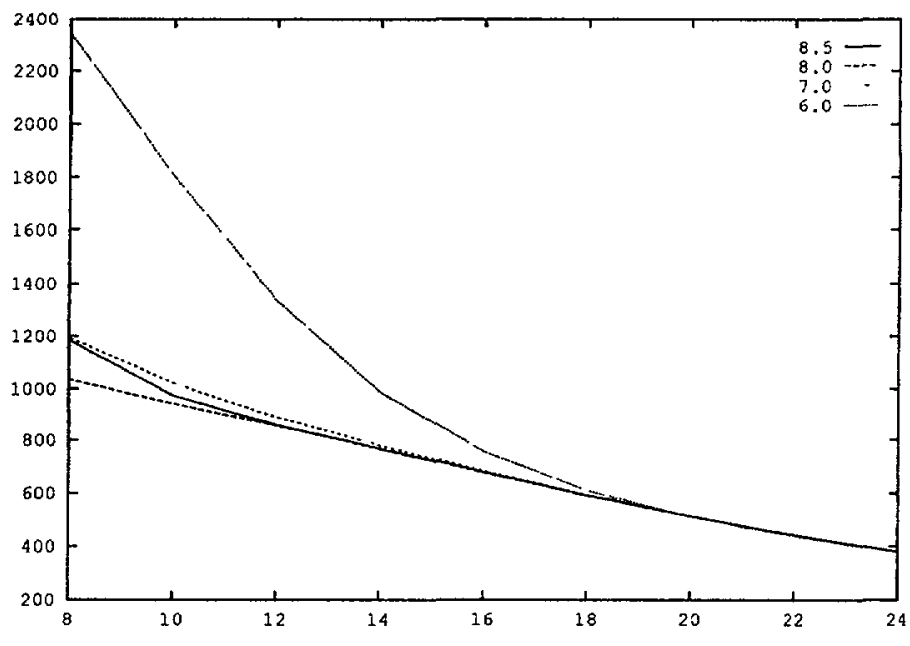

Fig. 2. - Ewald sum parameters: a) $V^{\text {ewald }}$ and b) $F^{\text {ewald }}=\sum\|\mathbf{f}\|^{2}$ for cutoff radius of 8.5, 8.0, 7.0 and $6.0 \AA$ as functions of $\nu$ where $\eta=\nu / L$, and $L$ is the box size.

Table III. - Lattrce constant.

\begin{tabular}{|c|c|c|}
\hline$T\left({ }^{\circ} \mathrm{C}\right)$ & Simulation $(\AA)$ & Experiment $(\AA)$ \\
\hline 325 & 7.68 & $7.522 \pm 0.002$ \\
420 & 7.75 & $7.58 \pm 0.01$ \\
\hline
\end{tabular}

accordance with experimental results. The streaks for $\beta \simeq 0$ and $\beta \simeq 180^{\circ}$ on Figure 3 are caused by the singularity inherent to Euler angle representation. Table III compares simulation and experimental lattice constants.

In order more precisely to compare the simulation with experiment, an expansion of the molecular orientational probability density function $P(\Omega)$ on a set of rotators functions $R_{\lambda \lambda^{\prime}}^{l}(\Omega)$ 


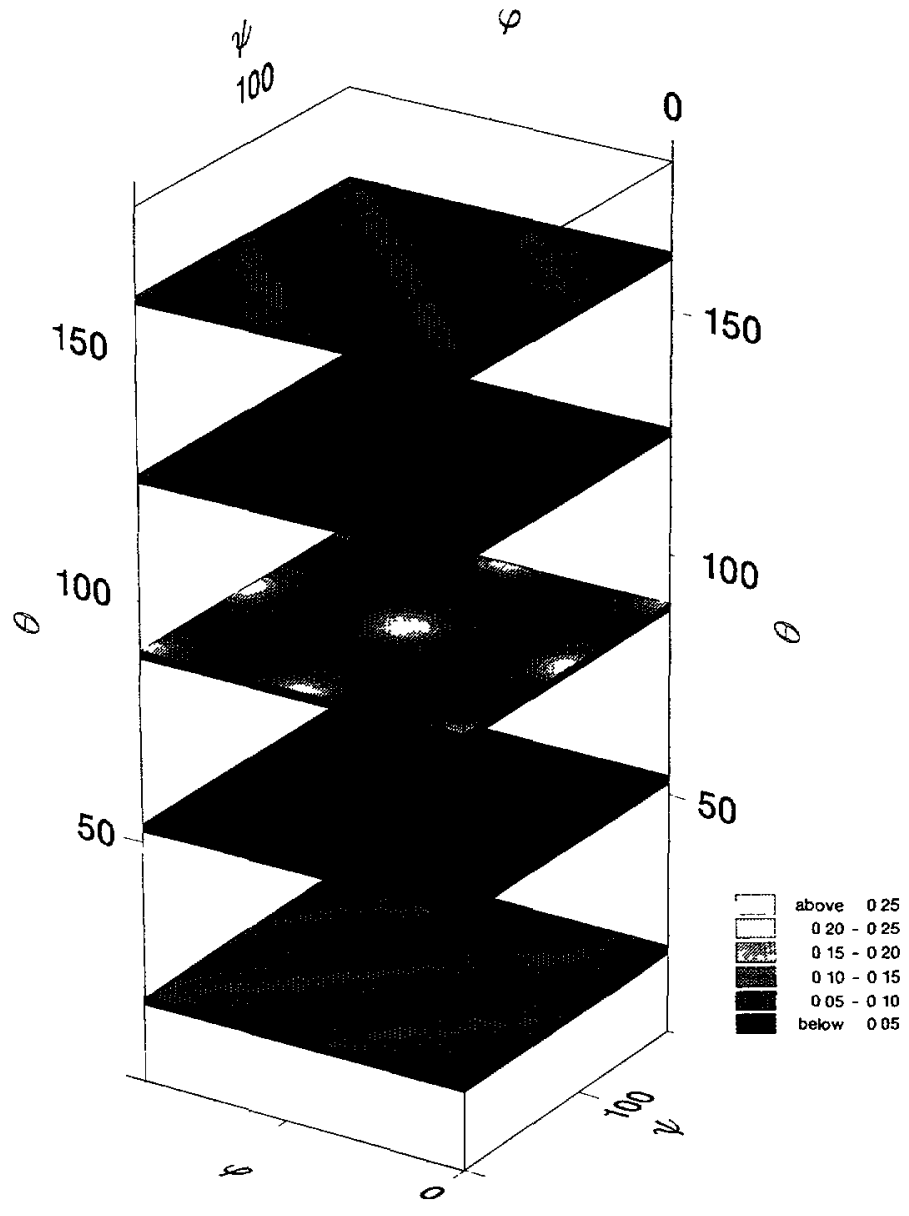

$600 \mathrm{~K}$

Fig. 3. - Orientational probability density function of the $\mathrm{ClO}_{4}$ ions, calculated over a $100 \mathrm{ps}$ run at $T=325{ }^{\circ} \mathrm{C}$, as a function of the Euler angles $(\Psi, \theta \varphi)$. The streaks for $\theta \sim 0$ (resp. $\theta \sim \pi$ ) are due to $\Psi$ and $\varphi$ (resp. $\Psi$ and $-\varphi$ ) being undistingurshable.

adapted to the symmetries of the ion and of the crystalline site was used [14]:

$$
P(\Omega)=\sum_{\ell, \lambda, \lambda^{\prime}} \frac{2 \ell+1}{8 \pi^{2}} A_{\lambda \lambda^{\prime}}^{\ell} R_{\lambda \lambda^{\prime}}^{\ell}(\Omega)
$$

where $A_{\lambda \lambda^{\prime}}^{\ell}$ denotes the coefficients of the expansion. Because of the invariance of $P(\Omega)$ with respect to the symmetry operations of the site group and of the molecular group, only few expansion coefficients $A_{\lambda \lambda^{\prime}}^{\ell}$ are non zero [15]. In the case of $\mathrm{ClO}_{4}^{-}$the first non zero coefficients are : $A_{11}^{4}, A_{11}^{6}, A_{11}^{8}, A_{11}^{10}$. The coefficient $A_{11}^{0}$ s taken to be equal to 1 due to the normalization condition of $P(\Omega)$. This allows to compute a structure factor that can be fitted to neutron diffraction results. Therefore, the first coefficients of the expansion and the two translational disorder terms, $\left\langle u^{2}\right\rangle_{\mathrm{K}}$ for potassium and $\left\langle u^{2}\right\rangle_{\mathrm{ClO}_{4}}$ for the perchlorate center of mass motion, were computed from: 
Table IV. - Comparison of the simulation and experimental results, using an expansion on a set of symmetry adapted functions at two temperatures. The experimental results were obtained by fitting the model to Bragg peak intensities. Bragg peaks were computed from the simulation and the model fitted to them, then the same parameters were computed directly. Had all the perchlorate ions been in perfect $T_{\mathrm{d}}$ orientations, all coefficients $A_{\lambda \lambda^{\prime}}^{\ell}$ would have been equal to 1

\begin{tabular}{|c|c|c|c|}
\hline$T=325^{\circ} \mathrm{C}$ & experiment & simulat.(Bragg) & simulat.(direct) \\
\hline$A_{11}^{4}$ & $0.44 \pm 0.03$ & $0.60 \pm 0.04$ & 0.21 \\
$A_{11}^{6}$ & $0.07 \pm 0.02$ & $-0.07 \pm 0.21$ & 0.14 \\
$A_{11}^{8}$ & $0.45 \pm 0.17$ & $0.95 \pm 1.06$ & 0.054 \\
$A_{11}^{10}$ & $0.10 \pm 0.18$ & $0.68 \pm 0.94$ & 0.03 \\
$\left\langle u_{x}^{2}\right\rangle_{\mathrm{K}^{+}}$ & $0.101 \pm 0.006$ & $0.101 \pm 0.016$ & 0.095 \\
$\left\langle u_{x}^{2}\right\rangle_{\mathrm{ClO}_{4}^{-}}$ & $0.093 \pm 0.002$ & $0.119 \pm 0.012$ & 0.115 \\
\hline
\end{tabular}

\begin{tabular}{|c|c|c|c|}
\hline$T=420^{\circ} \mathrm{C}$ & experiment & simulat.(Bragg) & simulat.(direct) \\
\hline$A_{11}^{4}$ & $0.41 \pm 0.03$ & $0.55 \pm 0.09$ & 0.16 \\
$A_{11}^{6}$ & $0.05 \pm 0.03$ & $0.03 \pm 0.40$ & 0.10 \\
$A_{11}^{8}$ & $0.36 \pm 0.20$ & $0.42 \pm 2.10$ & 0.03 \\
$A_{11}^{10}$ & $-0.04 \pm 0.24$ & $0.28 \pm 1.93$ & 0.02 \\
$\left\langle u_{x}^{2}\right\rangle_{\mathrm{K}^{+}}$ & $0.119 \pm 0.007$ & $0.123 \pm 0.030$ & 0.12 \\
$\left\langle u_{x}^{2}\right\rangle_{\mathrm{ClO}_{4}^{-}}$ & $0.110 \pm 0.003$ & $0.131 \pm 0.024$ & 0.14 \\
\hline
\end{tabular}

a) the neutron experiment,

b) the simulation, by computing same number of Bragg peak intensities as in the experiment, and then feeding them into the same model as if it were a real experiment, and,

c) the simulation, directly from the molecular positions and orientations.

These three sets of coefficients [16] allow to compare experimental and simulation results. By comparing a) and b) one checks the validity of the simulation, and by b)-c) comparison, the model used by the experimentalists. Table IV summarizes these results. In all cases the translational disorder as given by $\left\langle u_{x}^{2}\right\rangle_{K^{+}}$and $\left\langle u_{x}^{2}\right\rangle_{\mathrm{ClO}_{4}^{-}}$are consistent within a very good approximation.

The situation however is more complex for the orientational disorder. The expansion coefficients obtained from the Bragg peak fitting of the experiments and the simulation (a)-b) comparison), while relatively close at both temperatures at least for the lower values of $\ell$, are significantly weaker for the experiment than for the simulation. This means that the simulation is orientationaly less disordered than the real sample, since the situation in which all coefficients vanish to zero corresponds to isotropy. On the other hand, the directly computed coefficients are much weaker than the fitted ones (b) vs. c)). The orientations of the maxima. of the opdf are unaffected by these variations, therefore the most probable orientations are of $T_{\mathrm{d}}$ symmetry in all cases. However, this analysis shows that while the expansion method ylelds the correct symmetry, it is unreliable when quantitative results are required, presumably because of the small number of experimental data. 


\section{Orientation-Translation Correlations at $T=325{ }^{\circ} \mathrm{C}$}

Since the simulation reproduces reasonably well known crystallographic data, we may proceed to more sophisticated quantities. We wish to estimate the correlations between the set of variables $\left\{x_{1}, \ldots, x_{p}\right\}$ (e.g. molecular center-of-mass positions) and the set $\left\{x_{p+1} \ldots, x_{n}\right\}$ (e.g. molecular orientations). The correlation densities:

$$
C\left(x_{1}, \ldots, x_{p} ; x_{p+1}, \ldots, x_{n}\right)=P\left(x_{1}, \ldots, x_{p}, x_{p+1}, \ldots, x_{n}\right)-P\left(x_{1} \ldots, x_{p}\right) P\left(x_{p+1}, \ldots, x_{n}\right)
$$

where $P$ denotes probability density function of a set of variables $\left\{x_{\ell}\right\}$, are computed following the methods detailed in reference [8], and summarized in Appendix A.

\subsection{Single Molecule TO Correlations}

4.1.1. Symmetry. - The single molecule TO correlation density $C\left(u_{r} ; \eta, \zeta\right)$ was calculated for the perchlorate ion, $u_{r}$ being the molecular center of mass displacement projected on a direction $\mathbf{r}$ and $(\eta, \zeta)$ the polar angles that define the orientations of the perchlorate threefold symmetry axes, i.e. the $\mathrm{Cl}-\mathrm{O}$ bonds. It turns out to be an even function of $u_{r}$ for all $(\eta, \zeta)$ as apparent in Figure 4 for $\mathbf{r} / /[110]$. This 'expermental' fact is rather unexpected as the only constraint on $C\left(u_{r} ; \eta, \zeta\right)$ is that

$$
\int_{-\infty}^{+\infty} C\left(u_{r} ; \eta, \zeta\right) \mathrm{d} u_{r}=0
$$

- and the equivalent for $(\eta, \zeta)$ integration - which does not require any given symmetry. Indeed, an odd function of $u_{r}$ would seem natural, as it would simply displace slightly the maximum of the single molecule TO probability density $P\left(u_{r}, \eta, \zeta\right)$, meaning that for a given orentation $(\eta . \zeta)$, the molecular center of mass is moved by a certain quantity, resulting in a straightforward TO coupling situation. On the other hand, an even function ylelds a narrowing or a broadening of $P\left(u_{r}, \eta, \zeta\right)$, depending on whether the central part of the function is positive or negative, with no displacement. This seems reasonable when orientation $(\eta, \zeta)$ corresponds to a high symmetry direction of the crystal but is surprising in general orientation.

Following our results, however, the correlation density was fitted with the two parameter function:

$$
C\left(u_{r}: \eta, \zeta\right)=A_{\eta, \zeta}\left(1-2\left(\frac{u_{r}}{\gamma_{\eta, \zeta}}\right)^{2}\right) \exp \left[-\left(\frac{u_{r}}{\gamma_{\eta, \zeta}}\right)^{2}\right]
$$

which both is even and has the correct property of equation (6). This is not a model, but only a function with convenient properties. For a direction $(\eta, \zeta), A_{\eta \cdot \zeta}$ represents the magnitude of the correlations and $\gamma_{\eta, \zeta}$ is directly proportional to the molecular translational motion. When for a given orientation $(\eta, \zeta), A_{\eta, \zeta}>0$, it means that $C_{\eta, \zeta}>0$ for $-\frac{\gamma_{\eta, \zeta}}{\sqrt{2}}<u_{r}<+\frac{\gamma_{\eta \zeta}}{\sqrt{2}}$, that is, the molecular centers of mass tend to stay closer to their mean positions than if there were no TO correlation. Conversely. $A_{\eta, \zeta}<0$ yields two maxima at $\pm \sqrt{\frac{3}{2}} \eta \eta, \zeta$ resulting in a broadening of the translational distribution due to TO coupling.

4.1.2. $\mathrm{r} / /[110]$. - In the following, the pixel size $1515^{\circ} \times 15^{\circ}$ in the $(\eta, \zeta)$ plane. We now discuss the results for the projection direction $r / /[110]$, the first neighbour direction in the fcc lattice.

The fit of expression $(7)$ to $C\left(u_{r} ; \eta, \zeta\right)$, yields $A_{\eta, \zeta}$, shown on Figure 5 as a function of $\eta$ and $\zeta$. The elght maxima are for the molecular threefold axes parallel to the crystal threefold axes $(\eta \sim$ $55^{\circ}, \zeta=45^{\circ}$; .), i e. for $T_{\mathrm{d}}$ molecular orientations. Therefore, for these orientations, the center of mass translational displacements along the [110] direction tend to be smaller than suggested 


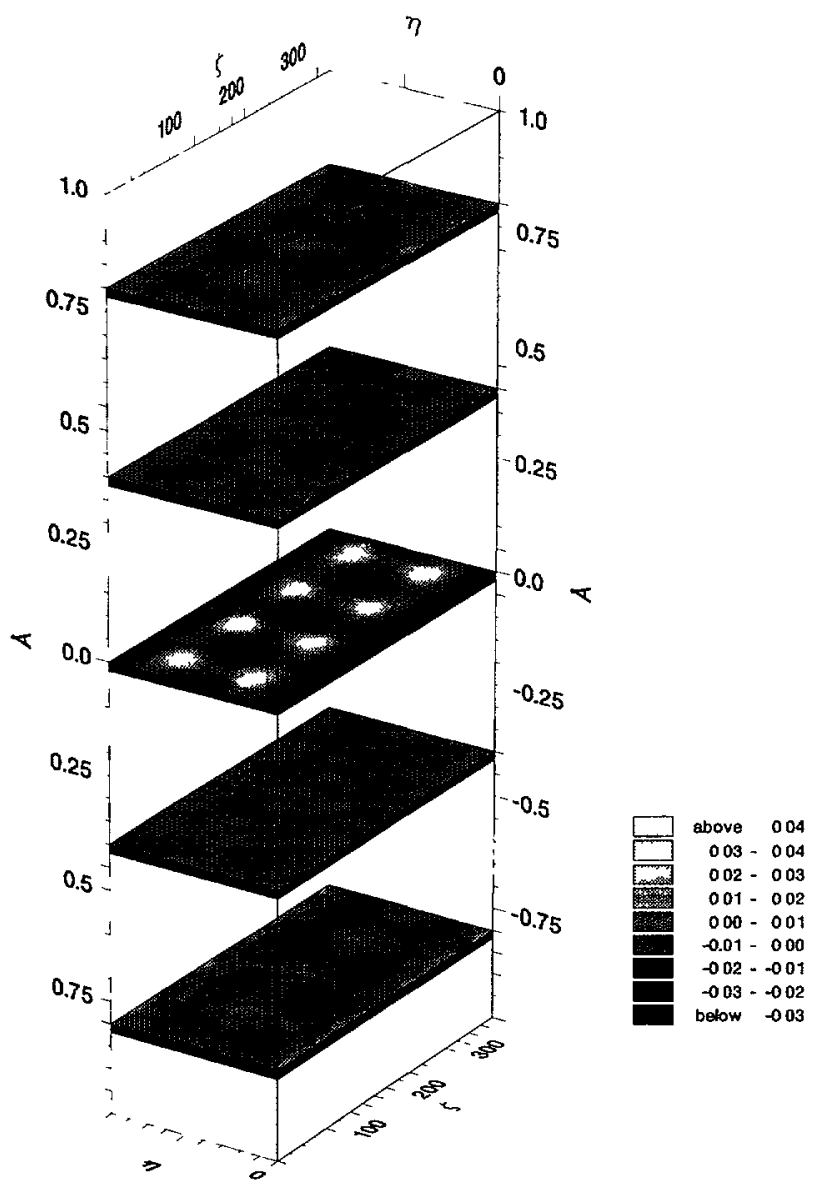

$C(u ; \eta, \zeta) 110$

Fig. 4. - Single molecule TO correlation, $C\left(u_{r} ; \eta, \zeta\right)$ with center of mass displacement u projected on the $[110]$ direction.

by the value of $\left\langle u_{r}^{2}\right\rangle_{\mathrm{ClO}_{4}}$. Indeed the halfwidth of the central peak is $\frac{\gamma_{\eta, \zeta}}{\sqrt{6}} \simeq 0.2 \AA$, when the parameter $A_{\eta, \zeta}$ is not too small, which is smaller than the translational disorder $\sqrt{\left\langle u_{r}^{2}\right\rangle_{\mathrm{ClO}_{4}}} \sim$ $0.3 \AA$ (cf Tab. 4). Elsewhere, in non $T_{\mathrm{d}}$ orlentations $\left(\eta=90^{\circ}, \zeta=90^{\circ}\right)$, where $A_{\eta, \zeta}<0$, the molecular centers of mass tend to undergo larger displacements of approximately $0.6 \AA$. We obtain therefore a narrowing of translational disorder in the most probable orientations of $T_{\mathrm{d}}$ symmetry, and a broadening due to TO coupling in non $T_{\mathrm{d}}$ orientations. The $\mathrm{ClO}_{4}$ lons are thus submitted to TO coupling that displaces them away from their mean center of mass position when in process of reorenting, the displacement distribution being symmetric with respect to the crystal site.

These results are quite different from those obtained using the same method in neopentane [8] for which TO coupling also takes place when the molecules are not in their mast probable 


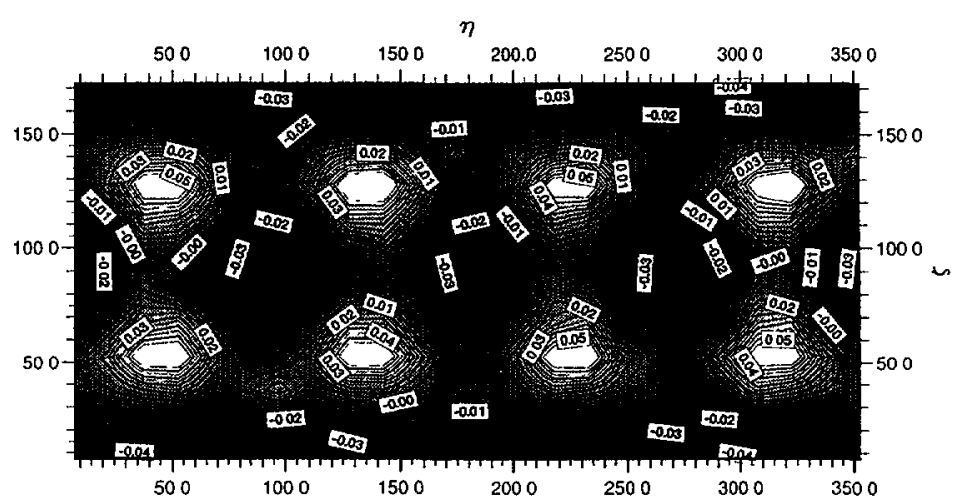

Fig. 5. - Angular dependence of the single molecule TO correlation amplitude $A_{\eta, \zeta}$ in the [110] direction using the expression in equation (7). The light grey areas correspond to positive correlations, the dark ones to negative correlations.

orientations (of $D_{2 d}$ symmetry for neopentane). In that case, however, $C\left(u_{r}, \eta, \zeta\right)$ is an odd function of $u_{r}$ meaning that the mean center of mass $\left(u_{r}=0\right)$ is an actual equilibrium position only when there is no TO correlation. In non-zero correlation situations, there is only one maximum, which should enable one in principle to trace the actual center of mass trajectory coupled to a reorientation. Conversely, in potassium perchlorate, when $A_{\eta, \zeta}<0$, we obtain two symmetric maxima, a degeneracy indicating that another parameter besides the $\mathrm{Cl}-\mathrm{O}$ bond orientations should be taken into account, presumably, those of the others bonds i.e. the complete molecular orientation, not simply that of one bond.

4.1.3. $\mathrm{r} / /[100]$. - The same analysis can be done for $\mathrm{r} / /[100]$, that is, the direction of the closest potassium ion. The results are similar, with smaller intensity. This is not trivial since the potassium ions are closer to the perchlorate molecules than the closest nelghbouring perchlorate. This property is probably due to the small size of the $\mathrm{K}^{+}$ion.

4.2. Two Molecule TO Correlatrons. - We now discuss two molecule correlations between the orientation of molecule 2 (always a perchlorate ion), and the center-of-mass translation molecule 1 (either a perchlorate ion with connecting vector $s_{1,2}=\left[\frac{1}{2}, \frac{1}{2}, 0\right]$, or a potassium lon, $\left.\mathrm{s}_{1,2}=\left[\frac{1}{2}, 0,0\right]\right)$.

4.2.1. Potassium-Perchlorate Coupling.. - Figure 6 represents the function $C\left(u_{1} ; \eta_{2}, \zeta_{2}\right)$ for a projection of the center of mass displacement $u_{1}$ of the potassium in the direction $\mathbf{r}=[100]$, and a perchlorate $\mathrm{Cl}-\mathrm{O}$ bond orientation $\left(\eta_{2}, \zeta_{2}\right)$. It clearly shows an odd function of $u_{1}$, so that an odd function was fitted to $C\left(u_{1} ; \eta_{2}, \zeta_{2}\right)$ :

$$
C\left(u_{r} ; \eta, \zeta\right)=A_{\eta, \zeta} \frac{u_{r}}{\gamma_{\eta, \zeta}} \exp \left[\frac{1}{2}\left(1-\left(\frac{u_{r}}{\gamma_{\eta, \zeta}}\right)^{2}\right)\right]
$$

If $A_{\eta, \zeta}>0$ then $C\left(u_{r} ; \eta, \zeta\right)$ is positive when $u_{r}$ 1s positive. The opposite holds for negative values. Figure 7 shows that $A_{\eta, \zeta}$ also has eight maxima in the crystal threefold axes directions, so that when a potassium ion moves toward a first neighbour perchlorate, the perchlorate tends to rotate toward a $T_{\mathrm{d}}$ orientation 1.e. when a potassium moves in the [100] direction toward a perchlorate, the latter gets orientationaly trapped, the $\mathrm{Cl}-\mathrm{O}$ bonds being forced to move away 


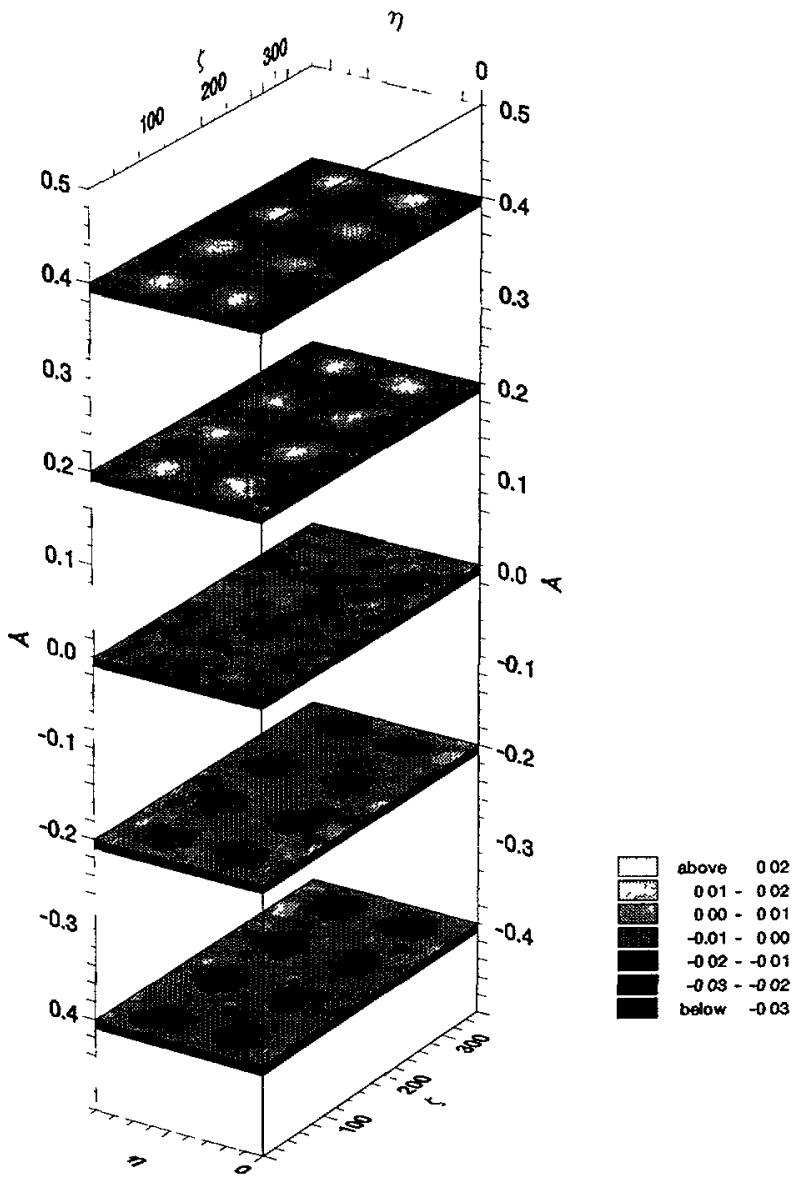

$C\left(u_{1} ; \eta_{2}, \zeta_{2}\right) 100$

Fig. 6. - Correlation density between the orientation of a perchlorate ion and the translation of a potassium lon: $C\left(u_{r_{1}} ; \eta_{2}, \zeta_{2}\right)$. The connecting vector is: $\mathbf{s}_{1,2}=\left[\frac{1}{2}, 0,0\right]$.

from the $[100]$ direction. The attractive electrostatic forces between the ions $\mathrm{K}^{+}$and $\mathrm{ClO}_{4}^{-}$ ( $q_{\mathrm{O}}=-0.25 e, q_{\mathrm{K}}=+e$ ) are completely negligible because, at this distance, it is the short range steric hindrance that is dominant. Conversely when the potassium ion moves away on the [100] direction, the $T_{d}$ orientation of the first neighbour perchlorate is not favored.

4.2.2. Perchlorate-Perchlorate Coupling.. - The analysis is the same as for potassium-perchlorate coupling but Figure 8 shows that $\left(\mathrm{K}^{+}-\mathrm{ClO}_{4}^{-}\right)$correlations are much stronger than $\left(\mathrm{ClO}_{4}^{-}-\mathrm{ClO}_{4}^{-}\right)$correlations, which can be explained by the shorter distance.

\section{Orientation-Orientation Correlations}

Clearly, for OO correlations, both molecules 1 and 2 are perchlorate ons and $s_{1,2}=\left[\frac{1}{2}, \frac{1}{2}, 0\right]$. Figure 9 shows the orientation-orientation correlation density $C\left(\alpha_{1} ; \alpha_{2}, \beta_{1,2}\right)$ where $\alpha_{1}$ (resp. $\alpha_{2}$ ) is the angle between the $\mathrm{Cl}-\mathrm{O}$ bond of molecule 1 (resp. 2) and the connecting vector 


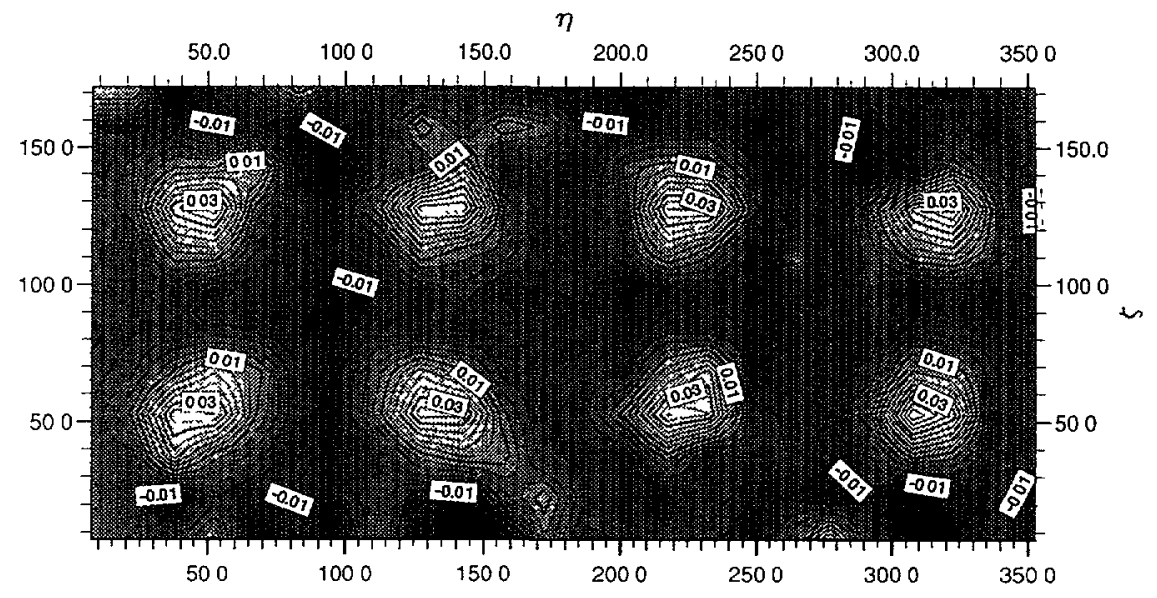

Fig. 7. - Amplitude of the correlation density between the orientation of a perchlorate ion and the translation of a potassium ion $A_{\eta, \zeta}$, using equation (8). The projection direction is [100]. The connecting vector is $s_{12}=\left[\frac{1}{2}, 0,0\right]$.

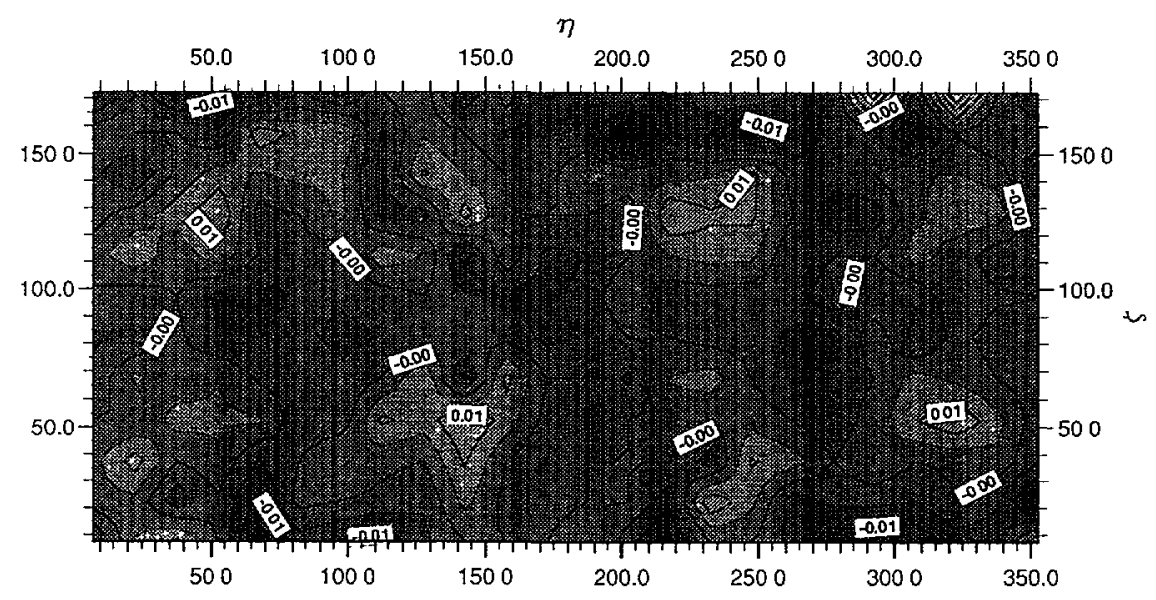

Fig. 8. - Angular dependence of the two molecule TO correlation amplitude $A_{\eta, \zeta}$ using equation (8), where the projection direction is [110]. The connecting vector $15 \mathbf{s}_{1,2}=\left[\frac{1}{2}, \frac{1}{2}, 0\right]$.

$\mathbf{s}_{1,2}$, and $\beta_{1,2}$ is the dihedral angle between the planes that contain $\mathbf{s}_{1,2}$ on the one hand, and the $\mathrm{Cl}-\mathrm{O}$ bonds of molecules 1 and 2 on the other [8]. The maxima visible on Figure 9 are compatible with the two molecules being in two opposite $T_{\mathrm{d}}$ orientations, e.g. the bond of molecule 1 are in [111], [i $\overline{1} 1],[1 \overline{1} \overline{1}]$ and [i11] while those of molecule 2 are in directions $[1 \overline{1} 1],[\overline{1} 11],[11 \overline{1}]$ and $[\overline{1} \overline{1} \overline{1}]$ (Fig. 10). The minima on Figure 9 on the other hand are compatible with the two molecules being in the same $T_{\mathrm{d}}$ orientation. This is similar to an antiferromagnetic coupling of spins. 


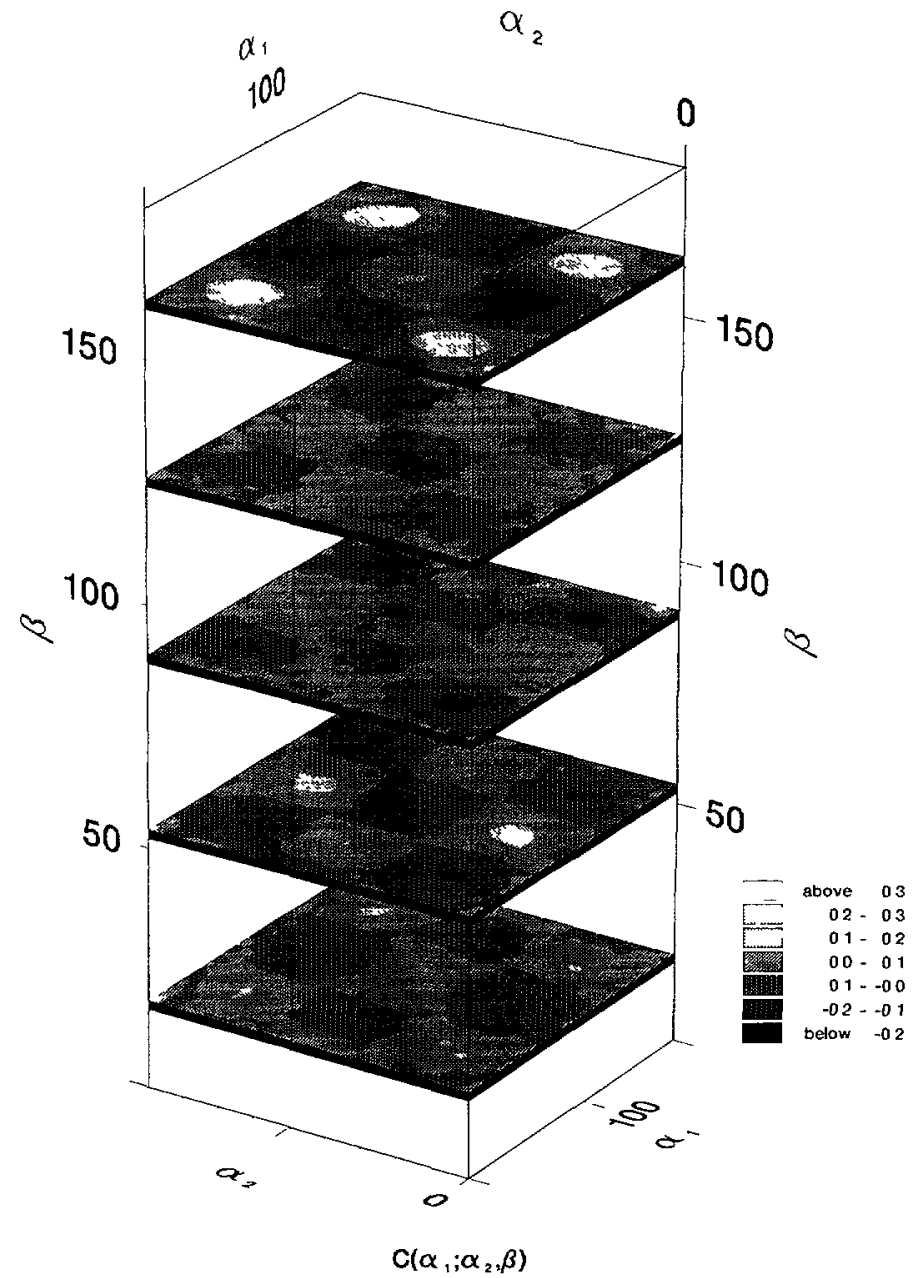

Fig. 9. - OO correlation density $C\left(\alpha_{1} ; \alpha_{2}, \beta_{1,2}\right)$, of two neighbourng perchlorate ions. The connecting vector is $\mathbf{s}_{1,2}=\left[\frac{1}{2}, \frac{1}{2}, 0\right]$.

5.1. PSEUdospin Model. - Thus, if $x, y$, and $z$ are the coordinates of the oxygen atoms in a reference frame whose axes are parallel to those of the crystal frame, but centered on the $\mathrm{Cl}$ atom of that ion, we define for each molecule a pseudospin $\sigma$ such that:

- if $(x>0, y>0, z>0),(x<0, y<0, z>0),(x<0, y>0, z<0)$ and $(x>0, y<0, z<$ 0 ), we assume that this molecule is in one of the $T_{\mathrm{d}}$ orlentations and write $\sigma=1$.

- If $(x>0, y>0, z<0),(x<0, y<0, z<0),(x<0, y>0, z>0)$ and $(x>0, y<0, z>$ $0)$, the molecule is in the other $T_{\mathrm{d}}$ orientations and write $\sigma=-1$.

- and if neither condition is verified, we assume that this molecule is in non- $T_{\mathrm{d}}$ orientation and write $\sigma=0$.

Table $\mathrm{V}$ shows $P(\sigma)$ at both temperatures. In an Arrhenius model, this yields an activation energy of approximately $530 \mathrm{~K}$ for the higher energy non- $T_{\mathrm{d}}$ state, a relatively low value [17]. 
Before and behind the plane $(x y)$

In the plane (xy)

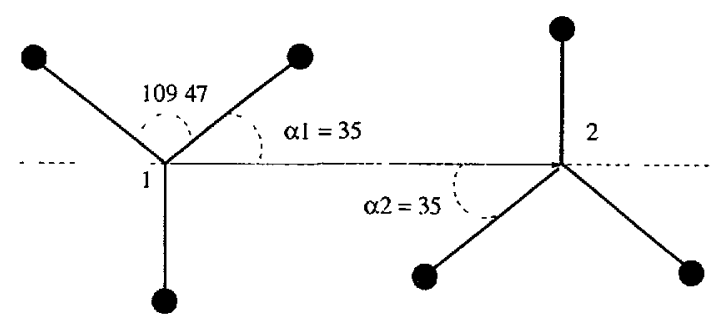

Fig. 10. - Two perchlorate ions in different $T_{d}$ orientations, an occurence which corresponds to the maxima of the $\mathrm{OO}$ correlation density $C\left(\alpha_{1} ; \alpha_{2}, \beta_{1,2}\right)$. This leads to the hypothesis of pseudospins with antiferromagnetic type coupling.

Table V. - $P(\sigma)$ for both simulation temperatures, where $\sigma$ is the pseudospin associated with $T_{\mathrm{d}}$ orientations. $T_{0}$ is given by: $P(\sigma=0)=\mathrm{e}^{\frac{-T_{0}}{T}}$.

\begin{tabular}{|c|c|c|c|}
\hline$T\left({ }^{\circ} \mathrm{C}\right)$ & -1 & 0 & 1 \\
\hline 325 & 0.29 & 0.42 & 0.29 \\
420 & 0.27 & 0.46 & 0.27 \\
\hline
\end{tabular}

With this definition, the $O O$ correlation can be written as a pseudospin-pseudospin correlation density:

$$
C\left(\sigma_{1} ; \sigma_{2}\right)=P\left(\sigma_{1}, \sigma_{2}\right)-P\left(\sigma_{1}\right) P\left(\sigma_{2}\right)
$$

Table VI gives $C\left(\sigma_{1} ; \sigma_{2}\right)$ for which $C(-1 ;-1)=C(+1 ;+1)=-C(-1 ;+1)=-C(+1 ;-1)$ corresponds to the antiferromagnetic coupling inferred from Figure 9 . Another way to see this is to calculate the usual correlation function:

$$
\left\langle\sigma_{1} \sigma_{2}\right\rangle=\sum_{\sigma_{1}, \sigma_{2}} C\left(\sigma_{1} ; \sigma_{2}\right) \sigma_{1} \sigma_{2}
$$

given that $\left\langle\sigma_{1}\right\rangle=\left\langle\sigma_{2}\right\rangle=0$. Using the results given in Table VI, this yields $\left\langle\sigma_{1} \sigma_{2}\right\rangle=-0.0185$ at $T=325^{\circ} \mathrm{C}$ and -0.0108 at $T=420{ }^{\circ} \mathrm{C}$, at the same time clearly negative and strongly temperature dependent.

The terms in Table VI that correspond to $\sigma=0$, 1.e. the middle line and column, show that there is very little, if any, correlation of $\sigma=0$ spins with $\sigma= \pm 1$ spins. Now, an antiferromagnetic structure on a cfc lattice should generate frustration, which the possibility for $\sigma=0$ however prevents in our case. An ordered, frustration-free structure could be constituted of a succession of planes with $\sigma= \pm 1$ and planes with $\sigma=0$, thus $P(\sigma=0)=0.5$, $P(\sigma=1)=P(\sigma=-1)=0.25$, and $\left\langle\sigma_{1} \sigma_{2}\right\rangle=-\frac{1}{6}$. While the probabilities are comparable to ours, the value taken by the correlation function is an order of magnitude greater. The situation in our simulation is thus that of a highly disordered system with a weak antiferromagnetic coupling. 
Table VI. - Correlation density of neighbouring pseudospins $C\left(\sigma_{1} ; \sigma_{2}\right) \times 10^{4}$.

\begin{tabular}{|c|c|c|c|}
\hline \multicolumn{4}{|c|}{$T=325^{\circ} \mathrm{C}$} \\
\hline$\sigma$ & -1 & 0 & 1 \\
\hline-1 & -43 & -4 & 47 \\
0 & -7 & 9 & -2 \\
1 & 50 & -5 & -45 \\
\hline
\end{tabular}

\begin{tabular}{|c|c|c|c|}
\hline \multicolumn{4}{|c|}{$T=420^{\circ} \mathrm{C}$} \\
\hline$\sigma$ & -1 & 0 & 1 \\
\hline-1 & -24 & -2 & 26 \\
0 & -6 & 4 & 2 \\
1 & 30 & -2 & -28 \\
\hline
\end{tabular}

Table VII. - Perchlorate reorientation time calculated from the average kinetic energy (first line) where $\Delta \eta=\pi / 2$, and $I$ is the moment of inertia, pseudospin residence time where: $\left\langle\sigma_{1}(0) \sigma_{1}(t)\right\rangle=\left\langle\sigma_{1}(0) \sigma_{1}(0)\right\rangle \exp \left(-t / \tau_{11}\right)$ (second line), and pseudospin correlation time where: $\left\langle\sigma_{1}(0) \sigma_{2}(t)\right\rangle=\left\langle\sigma_{1}(0) \sigma_{2}(0)\right\} \exp \left(-t / \tau_{12}\right)$ (third line), all given in picoseconds.

\begin{tabular}{|c|c|c|}
\hline$T$ & $325^{\circ} \mathrm{C}$ & $420^{\circ} \mathrm{C}$ \\
\hline$\tau_{0}=\Delta \eta \sqrt{\frac{I}{k_{\mathrm{b}} T}}$ & 0.65 & 0.60 \\
$\tau_{11}$ & 0.45 & 0.30 \\
$\tau_{12}$ & 0.65 & 0.55 \\
\hline
\end{tabular}

5.2. Correlated Orientational Dynamics.. - Using the pseudospin model, the orientational dynamics are easy to describe. Table VII gives some characteristic orientational times all of which are of the order of $0.5 \mathrm{ps}$. The first, $\tau_{0}$, is the time necessary to do a $90^{\circ}$ rotation, that is, the time it takes to go from a $\sigma=1$ to a $\sigma=-1$ state, or vice versa, with the average angular velocity due to temperature. The quantity $\left\langle\sigma_{1}(0) \sigma_{1}(t)\right\rangle$ can be computed as a function of time, and fitted with: $\left\langle\sigma_{1}(0) \sigma_{1}(0)\right\rangle \exp \left(-t / \tau_{11}\right)$ (Fig. 11). Thus, $\tau_{11}$ is the residence time

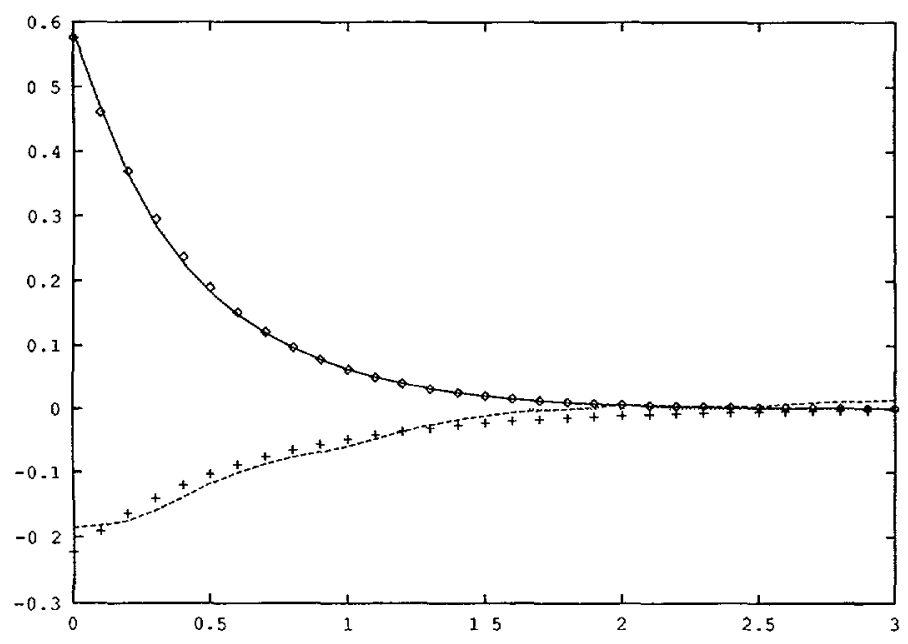

Fig. 11. - Orientational dynamics of the pseudospins at $T=325^{\circ} \mathrm{C} .\left\langle\sigma_{1}(0) \sigma_{1}(t)\right\rangle$ (line) fitted with $\left\langle\sigma_{1}(0) \sigma_{1}(0)\right\rangle \mathrm{e}^{\left(-t / \tau_{11}\right)}$ (open squares), and $\left\langle\sigma_{1}(0) \sigma_{2}(t)\right\rangle \times 10$ (line) fitted with $\left\langle\sigma_{1}(0) \sigma_{2}(0)\right\rangle \mathrm{e}^{\left(-t / \tau_{12}\right)}$ (crosses). 
of a spin in one of its \pm 1 states. $\tau_{0}$ is approximately the residence time in state $\sigma=0$, confirmed by the fact that $\tau_{0} / \tau_{11} \sim P(\sigma=0) / P(\sigma=1)$ as given in Table $\mathrm{V}$. In a similar fashion. $\left\langle\sigma_{1}(0) \sigma_{2}(t)\right\rangle$, yields $\tau_{12}$, the time during which two neighbourıng spins remain opposed. The noteworthy result is that $\tau_{12}>\tau_{11}$, which shows that molecular reorientations have, to some extent, a collective character, despite the weak coupling mentioned above. It appears that since the focus up to now in plastic crystal studies was on individual orientational motion (see e.g. the review paper [18]), it is the first time correlated orientational motion is observed. It would have been extremely difficult to show, had not the pseudospin model been valid. It is worth stressing, at this point, that this model was not chosen a proor, but arises naturally from our data.

\section{Summary and Conclusion}

The disordered phase of potassium perchlorate was simulated on a massively parallel computer with strict SIMD architecture. While it is difficult, for such applications, to use that architecture optimally, a large sample was simulated in a reasonable time.

The results are consistent with diffraction experiments, regarding lattice constant, translational disorder, and the symmetry of the most probable orientations of the perchlorate ions. The expansion of the molecular orientational probability density function that had been used to interpret the experiments was thoroughly tested and proved reliable for the translational disorder contribution but only for the main features of the orientational probability density function.

The orientation-translation correlations of the perchlorate ions show that when a molecule is in one of its two $T_{d}$ most probable orientations, its center-of-mass motion is reduced with respect to mean translational disorder, while non- $T_{\mathrm{d}}$ oriented molecules tend to show larger displacements. The TO correlation between the orientation of a $\mathrm{ClO}_{4}$ molecule and the translation of a neighbourmg perchlorate ion shows preference for molecular $T_{\mathrm{d}}$ orientations when the neighbouring ion moves closer to the molecule. The correlation between the orientation of a perchlorate ion and the translation of a potassium ion shows a simlar but weaker effect.

Finally, the orientational correlations between two neighbouring perchlorate molecules display a pseudospin type antiferromagnetic coupling. The study of the dynamics of these spins show that two neighbouring ions tend to remain orientationaly coupled longer than the individual residence time in one of the $\sigma= \pm 1$ states. This is the first, to our knowledge, evidence of collective orentational motion in such crystals.

\section{Acknowledgments}

The authors wish to acknowledge the use of the facilities of the Site Expérimental en Hyperparallélısme, ETCA, 16 bis Avenue Prieur de la Cote d'Or, 94114 Arcueil cedex, France, where the simulation was carried out.

Comments by Robert Pick are also gratefully acknowledged. 


\section{Appendix A}

\section{Correlation Densities}

We describe here, as an example, the computation of the correlation density $C(\mathbf{u} ; \Omega)$ where $\mathbf{u}$ is the molecular center-of-mass displacement with respect to the mean molecular position, and $\Omega$ is a set of Euler angles that yield the molecular orientation with respect to a crystal reference frame. This is a six-variable function which can be replaced by a much more convenient threevariable function:

$$
C\left(u_{r} ; \eta, \zeta\right)=P\left(u_{r}, \eta, \zeta\right)-P\left(u_{r}\right) P(\eta, \zeta)
$$

where $u_{r}=\mathbf{u} \cdot \mathbf{r}$ is the projection of $\mathbf{u}$ on a given direction $\mathbf{r}$, and $(\eta, \zeta)$ are the polar angles of a $\mathrm{Cl}-\mathrm{O}$ bond in the perchlorate ion.

The simulation yields the orientational probability density $P(\eta, \zeta)$ as a sum of Dirac $\delta$ functions:

$$
P(\eta, \zeta)=\frac{1}{N} \sum_{\imath=1}^{N} \frac{\delta\left(\eta-\eta_{2}\right)}{\sin \eta_{\imath}} \delta\left(\zeta-\zeta_{2}\right)
$$

where $N$ is the product of the number of bonds in one ion by the number of ions and the number of timesteps in the simulation. $\left(\eta_{2}, \zeta_{2}\right)$ are the polar angles of bond $\imath$.

We must however use pixels of finite size, and thus define a coarse-grained probability density:

$$
\begin{aligned}
\bar{P}(\eta, \zeta) & =\frac{\int_{\eta}^{\eta+\Delta \eta} \int_{\zeta}^{\zeta+\Delta \zeta} P(\eta, \zeta) \sin \eta \mathrm{d} \eta \mathrm{d} \zeta}{\int_{\eta}^{\eta+\Delta \eta} \int_{\zeta}^{\zeta+\Delta \zeta} \sin \eta \mathrm{d} \eta \mathrm{d} \zeta} \\
& =\frac{\nu_{o}(\eta, \zeta)}{(\cos \eta-\cos (\eta+\Delta \eta)) \Delta \zeta}
\end{aligned}
$$

where $\nu_{o}(\eta, \zeta)$ is the fraction of bonds for which $\eta \leq \eta_{2}<\eta+\Delta \eta, \zeta \leq \zeta_{2}<\zeta+\Delta \zeta$. Treating $P\left(u_{r}\right)$ and $P\left(u_{r}, \eta, \zeta\right)$ in a similar fashion, the coarse-gramed correlation density writes:

$$
\bar{C}\left(u_{r} ; \eta, \zeta\right)=\frac{\nu_{t o}\left(u_{r}, \eta, \zeta\right)-\nu_{t}\left(u_{r}\right) \nu_{o}(\eta, \zeta)}{(\cos \eta-\cos (\eta+\Delta \eta)) \Delta \zeta}
$$

with obvious notations, the subscript $o$ standing for orientational and $t$ for translational.

This method can be extended in a straightforward manner to other correlation densities.

\section{References}

[1] Johansson G.B. and Lindqvist O., Acta Crystallogr. B 33 (1977) 2918.

[2] Pistorius C.W.F.T., J. Phys. Chem. Soluds 31 (1970) 385.

[3] Denise B., Debeau M., Depondt Ph. and Heger G., J. Phys. France 49 (1988) 1203.

[4] Allen M.P. and Tildesley D.J., Computer Simulations of Liquids (Oxford Science publ., 1990).

[5] Kilein M., McDonald I.R. and Ozaki Y., J. Chem. Phys. 79 (11) (1983) 5579.

[6] Heyes D.M., J. Chem. Phys. 74 (3) (1981) 1924.

[7] Ding H., Karasawa N. and Goddard W., J. Chem. Phys. 97 (6) (1992) 4309.

[8] Depondt Ph. and Breymann W., to be published in Molec. Phys.. 
[9] Rousseau B., Boutin A., Fuchs A.H. and Craven C.J., Molec. Phys. 76, (1992) 1079.

[10] Thinking Machine Corporation, Connection Machine CM-2 Technical Summary (Cambridge, MA, 1991).

[11] The denomination NEWS is slightly misleading since it seems to imply four neighbours, whereas, if all 16384 processors are used, the number of neighbours is 28 .

[12] Nosé S. and Klein M.L., Mol. Phys. 50 (1983) 1055.

[13] Rycerz Z.A. and Jacobs P.W.M., Molecular Simulation 8 (1992) 197.

[14] Press W. and Hüller A., Acta Crystallogr. A 29(1973) 252.

[15] Yvinec M. and Pick R.M., J. Phys. France 41 (1980) 1045.

[16] Breymann W., Depondt Ph. and Pick R., Can. J. Chem. 66 (1988) 798.

[17] Galam S. and Depondt Ph., Europhys. Lett. 5 (1988) 43.

[18] Meyer M. and Hardouin Duparc O.B.M., Adv. Solid State Chem. 3 (1993) 1. 